\title{
Galactic Winds and the Missing Baryons
}

\author{
Sylvain Veilleux ${ }^{\mathrm{A}}$ and David S. Rupke $\mathrm{A}, \mathrm{B}$ \\ ${ }^{\text {A }}$ Department of Astronomy, University of Maryland, College Park, MD 20740, USA \\ ${ }^{\text {B }}$ Corresponding author. Email: drupke@ astro.umd.edu
}

Received 2004 May 3, accepted 2004 September 3

\begin{abstract}
Galaxy-scale winds are poised to solve a number of ailments in modern cosmology. However, we know little about trends of wind properties with host galaxy properties. In an attempt to quantify the impact of galactic winds on their host galaxies and the environment, we are conducting a systematic survey of star-forming and active galaxies at $z=0-0.5$. Preliminary results on the star-forming galaxies are presented.
\end{abstract}

Keywords: galaxies: starburst — galaxies: active — galaxies: ISM — galaxies: kinematics and dynamics — intergalactic medium

\section{Galactic Winds and Cosmology}

In recent years, mechanical and radiative feedback from star formation and active galactic nuclei has been hailed as the 'miracle cure' to cosmology's ailments. This feedback may help to:

1 . Explain why (a) $\Omega_{\text {stars }} / \Omega_{\text {baryons }} \ll 1$ (Peebles 2004 ; Fukugita et al. 1998), and why (b) up to half of the baryons inferred to have once been in our Galaxy have not yet been detected (Silk 2003). This is the so-called baryon deficit. Winds may have inhibited early star formation and ejected a significant fraction of the baryons originally belonging to the Galaxy.

2. Suppress the number of visible dwarf galaxies (Moore et al. 1999). Their small escape velocities make them particularly vulnerable to galactic winds.

3. Avoid the 'cooling catastrophe' at high redshift that results in the overproduction of massive luminous galaxies (Cole et al. 2000). Feedback may heat the ISM of protogalaxies.

4. Remove material with low specific angular momentum early on (to thereby help enlarge gas disks in CDM simulations; Steinmetz \& Navarro 1999; Bullock et al. 2001; Binney et al. 2001). Galactic winds emerge from the centre of gravitational potentials, entraining material with low specific angular momentum in their path.

5. Explain the mass-metallicity relation in galaxies (Bregman 1978; Kauffmann \& Charlot 1998; van den Bosch 2002). Massive galaxies with deep gravitational potentials retain a larger fraction of their metals than dwarf galaxies.

6. Reduce the dark matter concentrations in galaxies by ejecting a significant fraction of their central baryons (Navarro et al. 1997; van den Bosch \& Swaters 2001; Binney et al. 2001).

7. Explain (a) the break between low- and hightemperature clusters in the correlation between their
$\mathrm{X}$-ray luminosities and temperatures, and (b) the lack of cluster evolution out to $z \sim 1$, by creating an entropy floor through radiative pre-heating and ejection of hot material from proto-galaxies (Kaiser 1991; Evrard \& Henry 1991; Lloyd-Davies et al. 2000; Borgani et al. 2002; Cavaliere et al. 2002).

8. Inhibit cooling flows in galaxy clusters with active $\mathrm{cD}$ galaxies (Böhringer et al. 1993; Fabian et al. 2000; McNamara et al. 2000, 2001; Blanton et al. 2002).

9. Enrich the ICM and IGM without significantly affecting the kinematics of the $\mathrm{Ly} \alpha$ forest (Renzini 1997; Buote 2000; Madau et al. 2001).

\section{Observational Context}

Given the many cosmological implications of galactic winds, we are clearly motivated to constrain their frequency of occurrence and empirical properties. Starburstdriven superwinds are common in a range of galaxy types: $\mathrm{L}^{*}$ or brighter galaxies with modest-to-high star formation rates (SFRs, $\Sigma_{*} \gtrsim 0.1 M_{\odot} \mathrm{yr}^{-1} \mathrm{kpc}^{-2}$; Lehnert \& Heckman 1995, 1996; Heckman et al. 2000; Rupke et al. 2002; Adelberger et al. 2003; Shapley et al. 2003); more quiescent galaxies like the Milky Way (Bland-Hawthorn \& Cohen 2003); and dwarf starbursting galaxies with $\Sigma_{*} \gtrsim 10^{-3} M_{\odot} \mathrm{yr}^{-1} \mathrm{kpc}^{-2}$ (e.g. Martin 1998). Observations have also shown that the empirical properties of starburst-driven winds vary over a large dynamic range: opening angles $\Omega / 4 \pi \sim 0.1-0.5$; maximum radii of a few$50+\mathrm{kpc}$; maximum velocities $\Delta v \sim 25-1000+\mathrm{km} \mathrm{s}^{-1}$; energies of $10^{53}-10^{57}$ ergs (about $1 / 10$ of the kinetic energy returned to the ISM by the starburst); and mass entrainment efficiencies $\eta \equiv(\mathrm{d} M / \mathrm{d} t) / \mathrm{SFR} \sim 0.01-5$.

Despite our increasing knowledge of galaxy-scale winds and the precision with which we can measure their properties, the relationships between the frequency of occurrence and properties of superwinds and the properties of the galaxies hosting these winds have 
yet to be established quantitatively. Such knowledge is important as the input to simulations of galaxy formation and evolution that incorporate such winds. Furthermore, superwinds have not been studied extensively outside of the local universe (except at very high redshift). Finally, we know little about winds in the most massive galaxies with the highest star formation rates.

\section{A Comprehensive Survey}

We are conducting a large survey of starburst-driven superwinds in over 75 galaxies, searching for statistical trends in wind properties as a function of host galaxy properties and redshift (Rupke et al. 2002; Rupke, Veilleux, \& Sanders, in preparation). Our sample consists of a large number of interacting galaxies with high star formation rates (ultraluminous infrared galaxies, ULIRGs) and redshifts up to $z \sim 0.5$, as well as a similar number of galaxies with lower SFR (leading to a range in SFR of two orders of magnitude).

To probe the properties of these winds, we use absorption-line spectroscopy of the NaI D $\lambda \lambda 5890,5896$ doublet, which is conveniently located below $1 \mu \mathrm{m}$ for galaxies with modest redshifts. This feature can be fitted with complex profiles to determine the outflow velocity $\Delta v$ and mass outflow rate $\mathrm{d} M / \mathrm{d} t$ for individual galaxies. Normalising the mass outflow rate to the global star formation rate then yields a measure of the amount of interstellar gas entrained in the wind relative to the gas turned into stars (the mass entrainment efficiency, $\eta$ ).

We divide our full sample into three parts to better compare galaxy types: (1) a sample of infrared-selected galaxies (IRGs) with $\langle z\rangle=0.03$ and $\langle$ SFR $\rangle=40 M_{\odot} \mathrm{yr}^{-1}$; (2) a 'low- $z$ ' ULIRG sample with $\langle z\rangle=0.13$ and $\langle\mathrm{SFR}\rangle=225 M_{\odot} \mathrm{yr}^{-1}$; and (3) a 'high- $z$ ' ULIRG sample with $\langle z\rangle=0.36$ and $\langle$ SFR $\rangle=389 M_{\odot} \mathrm{yr}^{-1}$.

\section{Results}

\subsection{Detection Rate}

We use a velocity cutoff of $-50 \mathrm{~km} \mathrm{~s}^{-1}$ to delineate galaxies with superwinds. We find that superwinds are more common in ULIRGs $(63 \pm 8 \%)$ than in galaxies with lower star formation rates $(43 \pm 8 \%)$; see Figure 1. However, our low- $z$ and high- $z$ ULIRG subsamples also show strikingly different frequencies of occurrence $(73 \pm 8 \%$ versus $38 \pm 13 \%)$. This could be interpreted as evidence for redshift evolution in these sources; however, it is more likely to be due to the difference in SFR between the two samples. The median SFR for the high- $z$ ULIRGs is twice that for the the low- $z$ ULIRGs, meaning galaxies with the highest star formation rates may be less able to produce massive winds.

\subsection{Velocities and Escape Fraction}

The observed distribution of NaI D velocity components (relative to systemic) is skewed toward the blue. The projected 'maximum' velocities in the components that

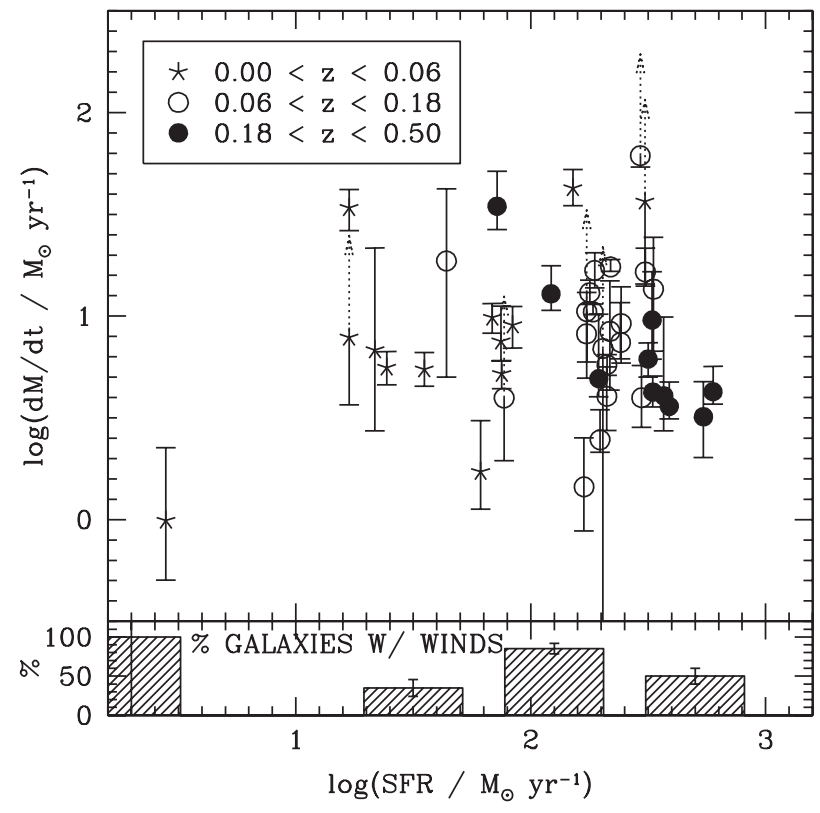

Figure 1 Mass outflow rate and detection rate as a function of star formation rate. There is no dependence of $\mathrm{d} M / \mathrm{d} t$ on SFR over this range, but the detection rate peaks at a few hundred $M_{\odot} \mathrm{yr}^{-1}$.

are outflowing (equal to the centroid velocity plus onehalf the velocity width) are $300-400 \mathrm{~km} \mathrm{~s}^{-1}$ on average, with a maximum of $\sim 600 \mathrm{~km} \mathrm{~s}^{-1}$ (though we do observe velocities up to $1200 \mathrm{~km} \mathrm{~s}^{-1}$ in one galaxy).

These velocities are smaller than or comparable to the escape velocity of a galaxy with $v_{\mathrm{c}}=200-300 \mathrm{~km} \mathrm{~s}^{-1}$, assuming an isothermal potential with $r_{\max } / r=10$. Ignoring halo drag (Silich \& Tenorio-Tagle 2001) or acceleration of the wind, we compute that at most $20 \%$ of the material in these winds will escape the galaxy and enter the IGM. However, the hot gas in these winds (carrying much of the metallicity) will be more likely to escape.

The host galaxy property that is the most straightforward to compute for our sample is the star formation rate (computed from $L_{\mathrm{IR}}$ ). In Figure 2 we plot $\Delta v$ as a function of SFR. Velocity in these galaxies is independent of star formation rate (and $K^{\prime}$-band magnitude, data not shown). This lack of a correlation extends over at least two orders of magnitude in star formation rate and almost four mag in $M_{K^{\prime}}$. The latter may trace the old stellar mass in these galaxies, but there is also an uncertain contribution from warm dust emission and young supergiants.

\subsection{Mass Outflow Rate}

To calculate the instantaneous mass outflow rate in the wind, we assume a spherically symmetric massconserving free wind, with $\mathrm{d} M / \mathrm{d} t$ and velocity independent of radius (see Rupke et al. 2002). We find that values of $\mathrm{d} M / \mathrm{d} t$ are similar across our entire dataset, ranging up to $50 M_{\odot} \mathrm{yr}^{-1}$ but mostly falling in the range 5$20 M_{\odot} \mathrm{yr}^{-1}$. In Figure 1, we also show that mass outflow rate in these galaxies is independent of star formation rate (and $K^{\prime}$-band magnitude, data not shown). 


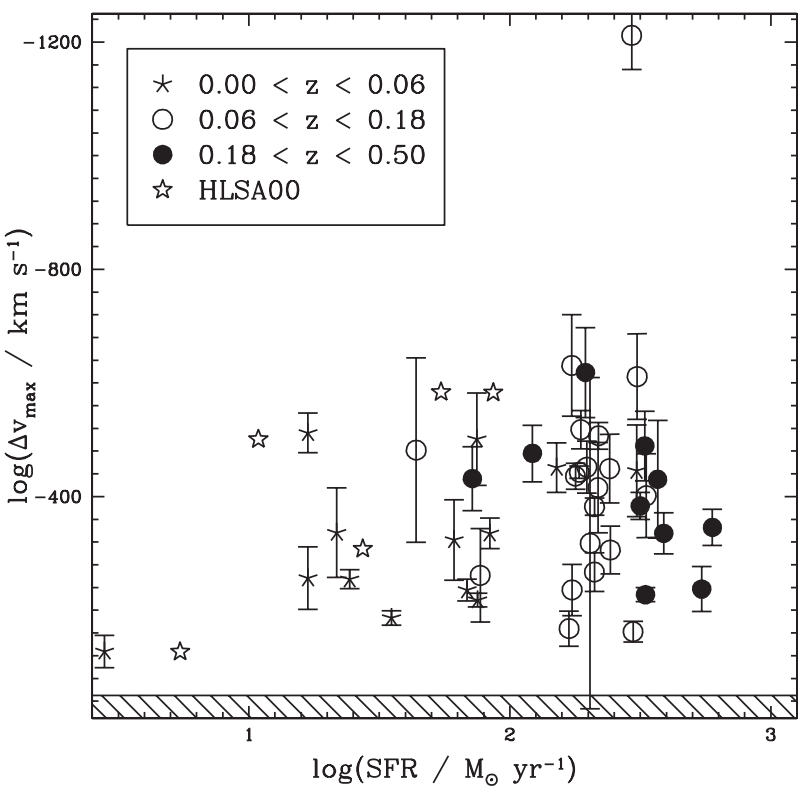

Figure 2 Terminal velocity as a function of star formation rate. There is no dependence of $\Delta v_{\max }$ on SFR over this range. The open stars are from Heckmann et al. (2000).

To explain the baryons 'missing' from the Galaxy (Silk 2003), which has a mass of order a few $10^{11} M_{\odot}$, a wind with $\mathrm{d} M / \mathrm{d} t=10 M_{\odot} \mathrm{yr}^{-1}$ would have to operate for a Hubble time and have an escape fraction of $100 \%$. Thus, the wind would have to be significantly more massive than we measure and have a greater chance of escaping the galaxy. Some of this mass could show up in other gas phases of the wind, the hottest of which is most likely to escape. The same argument can be applied to ULIRGs, which have dynamical masses of order $10^{11} M_{\odot}$ (Tacconi et al. 2002).

\subsection{Mass Entrainment Efficiency}

Previous measurements of $\eta$ in nearby dwarf galaxies, edge-on spirals, and nearby infrared-luminous galaxies indicate that this ratio is generically of order unity (Martin 1999; Heckman et al. 2000). However, we find a mean $\eta$ in LIRGs and ULIRGs that is smaller than this, in the range $0.01-0.1$, and that $\eta \sim \mathrm{SFR}^{-1}$.

The dependence of $\eta$ on SFR means that starbursts in ULIRGs are much less efficient in powering massive superwinds than those in LIRGs and less-luminous galaxies. What is the cause of this difference? One obvious possibility is the large concentrations of molecular gas in these galaxies (Sanders et al. 1991), which may obstruct the winds. Secondly, radiative losses are likely to be significant in the denser environment of ULIRGs (e.g. Downes \& Solomon 1998), therefore reducing the amount of energy available to drive the outflow.

\section{Outlook}

To further understand the dependence of outflow properties on host galaxy characteristics, this technique should be applied to samples of galaxies that are not infraredselected; moderate-resolution multi-object spectrographs would make this efficient. To probe the neutral phase of outflows more directly, absorption studies in Hi using sensitive dishes and broad-band correlators would be useful.

A complete description of these data is forthcoming (Rupke, Veilleux, \& Sanders, in preparation), and the results derived from our sample of star-forming galaxies will be compared with those found in a large sample of infrared-luminous AGNs (Rupke, Veilleux, \& Sanders, in preparation).

\section{Acknowledgments}

The authors acknowledge partial support from NSF/ CAREER grant AST-9874973 and NASA/LTSA grant NAG 56547.

\section{References}

Adelberger, K. L., et al. 2003, ApJ, 584, 45

Binney, J., Gerhard, O., \& Silk, J. 2001, MNRAS, 321, 471

Bland-Hawthorn, J., \& Cohen, M. 2003, ApJ, 582, 246

Blanton, E. L., Sarazin, C. L., \& McNamara, B. R. 2002, ApJ, 585,227

Böhringer, H., et al. 1993, MNRAS, 318, L25

Borgani, S., et al. 2002, MNRAS, 336, 409

Bregman, J. N. 1978, ApJ, 224, 768

Bullock, J. S., et al. 2001, ApJ, 555, 240

Buote, D. A. 2000, ApJ, 539, 172

Cavaliere, A., Lapi, A., \& Menci, N. 2002, ApJ, 581, L1

Cole, S., et al. 2000, MNRAS, 319, 168

Downes, D., \& Solomon, P. M. 1998, ApJ, 507, 615

Evrard, A. E., \& Henry, J. P. 1991, ApJ, 383, 95

Fabian, A. C., et al. 2000, MNRAS, 318, L65

Fukugita, M., Hogan, C. J., \& Peebles, P. J. E. 1998, ApJ, 503, 518

Heckman, T. M., et al. 2000, ApJS, 129, 493

Lehnert, M. D., \& Heckman, T. M. 1995, ApJS, 97, 89

Lehnert, M. D., \& Heckman, T. M. 1996, ApJ, 462, 651

Lloyd-Davies, E. J., Ponman, T. J., \& Cannon, D. B. 2000, MNRAS, 315,689

Kaiser, N. 1991, ApJ, 383, 104

Kauffmann, G., \& Charlot, S. 1998, MNRAS, 297, L23

Madau, P., Ferrara, A., \& Rees, M. J. 2001, ApJ, 555, 92

Martin, C. L. 1998, ApJ, 506, 222

Martin, C. L. 1999, ApJ, 513, 156

McNamara, B. R., et al. 2000, ApJ, 534, 135

McNamara, B. R., et al. 2001, ApJ, 562, L149

Moore, B., et al. 1999, ApJ, 524, L19

Navarro, J. F., Frenk, C. S., \& White, S. D. M. 1997, ApJ, 490, 493

Peebles, P. J. E. 2004, PASA, 21, 385

Renzini, A. 1997, ApJ, 488, 35

Rupke, D. S., Veilleux, S., \& Sanders, D. B. 2002, ApJ, 570, 588

Sanders, D. B., Scoville, N. Z., \& Soifer, B. T. 1991, ApJ, 370, 158

Shapley, A. E., et al. 2003, ApJ, 588, 65

Silich, S., \& Tenorio-Tagle, G. 2001, ApJ, 552, 91

Silk, J. 2003, MNRAS, 343, 249

Steinmetz, M., \& Navarro, J. F. 1999, ApJ, 513, 555

Tacconi, L. J., et al. 2002, ApJ, 580, 73

van den Bosch, F. C. 2002, MNRAS, 332, 456

van den Bosch, F. C., \& Swaters, R. A. 2001, MNRAS, 325, 1017 